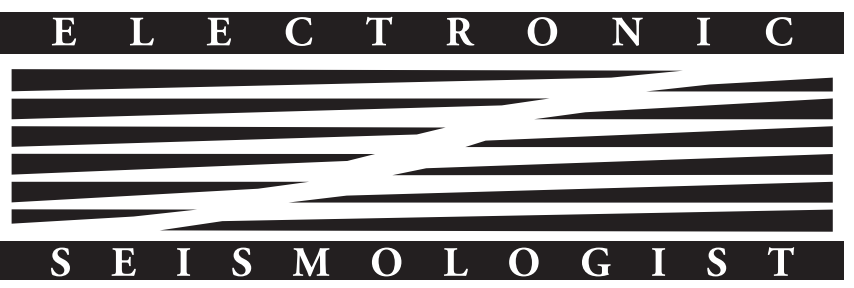

\section{Standards for Documenting Finite-Fault Earthquake Rupture Models}

\author{
by P. Martin Mai, Peter Shearer, Jean-Paul \\ Ampuero, and Thorne Lay
}

\section{ABSTRACT}

In this article, we propose standards for documenting and disseminating finite-fault earthquake rupture models, and related data and metadata. A comprehensive documentation of the rupture models, a detailed description of the data processing steps, and facilitating the access to the actual data that went into the earthquake source inversion are required to promote follow-up research and to ensure interoperability, transparency, and reproducibility of the published slip-inversion solutions. We suggest a formatting scheme that describes the kinematic rupture process in an unambiguous way to support subsequent research. We also provide guidelines on how to document the data, metadata, and data processing. The proposed standards and formats represent a first step to establishing best practices for comprehensively documenting input and output of finitefault earthquake source studies.

\section{INTRODUCTION}

Finite-fault source inversions have become a fundamental seismological tool to estimate the kinematic properties of earthquake rupture processes. Using seismic ground-motion recordings often augmented with geodetic and/or tsunami measurements, these inversions provide models of the space-time evolution of coseismic displacement that occurs on one or more fault segments. The resulting finite-fault rupture models (also known as slip models) quantify the spatial distribution and direction of slip on the fault, how fast the rupture expanded over the fault surface, and how long each point on the fault slipped. If only geodetic static displacements are used, the source inversions can constrain the fault geometry and static slip distribution (i.e., final displacements over the fault surfaces), but not the temporal rupture evolution. For large tsunamigenic earthquakes, tide-gauge records, or deep-water buoy recordings of sea-level variation may also be used, exclusively or in combination with other datasets, to quantify the rupture process with or without time information.

Kinematic source parameters from finite-fault models (fault-slip magnitude and slip-direction angle, rupture speed, rise time, and slip-rate function) are extensively utilized as input data for further seismological research, for example, to compute stress drop on the fault, static coulomb stress changes on surfaces in the surrounding medium (e.g., Stein, 2003), and stress interactions with postseismic processes (e.g., Ergintav et al., 2009); to infer dynamic-rupture behavior (e.g., Heaton, 1990; Ide and Takeo, 1997; Tinti et al., 2005; Mai et al., 2006; Causse et al., 2013), and aspects of rupture nucleation and complex propagation properties (e.g., Mai et al., 2005; Gabriel et al., 2012); to study earthquake mechanics and earthquake scaling (e.g., Mai and Beroza, 2000; Manighetti et al., 2005; Strasser et al., 2010); and to develop models that quantify rupture complexity for ground-motion simulations (e.g., Somerville et al., 1999; Mai and Beroza, 2002; Lavallée et al., 2006; Causse et al., 2010; K. K. S. Thingbaijam and P. M. Mai, unpublished manuscript, 2016, see Data and Resources). Slip distributions also play a role in understanding earthquake recurrence and aftershock distributions. As such, finite-fault rupture models provide a valuable resource to investigate and better understand earthquake source processes, which ultimately guide improved seismic-hazard analysis.

This contribution to the Electronic Seismologist proposes standards for documenting and disseminating finite-fault earthquake rupture models. Comprehensive documentation and timely dissemination of these models facilitates followup research and ensures interoperability, transparency, and reproducibility of the published slip-inversion solutions. Reproducible research is a key to advancing science in general, and to ensuring the credibility of our work. Recently, there has been extensive focus on reproducibility of research results, extending across computer science (Mesirov, 2010; Peng, 2011), medical science (Eddy et al., 2012), and geoscience (Agnew, 2012, 2013; Lees, 2012), including the issue of how to make valuable scientific data and results accessible (Hanson et al., 2011; Haak et al., 2012). In this context, it is interesting to note that the Stanford Exploration Project (see Data and Resources) has ensured reproducibility of their research products (Schwab et al., 2000) since 1991 by generating electronic 
reproducible documents as their principal means of technology transfer.

In addition to making the results of finite-fault inversions available to future users in a standardized format, we propose that all relevant metadata should also be published and ideally be made electronically accessible. The key here is to facilitate, not so much replicability, but rather reproducibility of the scientific work. In the former case, an experiment (inversion) is conducted under precisely the same conditions at different times, whereas the latter involves similar, but not identical, experiments (inversions) at different times, in different locations, and under somewhat different conditions (e.g., by different people, using different algorithms or compilers). To facilitate this, we advocate that sufficient metadata be provided as part of any published finite-fault slip model, be it in a journal publication or online.

Furthermore, we propose a formatting scheme that tabulates the kinematic rupture process in an unambiguous way to support subsequent scientific use of this data. The existing SRCMOD database of finite-fault rupture models (see Data and Resources, Mai and Thingbaijam, 2014) has been compiled by collecting data from individual authors for over a decade, tediously gathering needed metadata to provide at least the minimum supporting information about the inversion parameterization and the resulting source models. However, there are many more published rupture models that are not yet available in this database. In other cases, metadata is missing or incomplete, making reproducibility of these studies infeasible. A common and agreed upon standard for documenting and disseminating finite-fault rupture models will greatly facilitate not only the expansion and completion of such a global database, but also future seismological research on earthquake source processes. For instance, numerous rupture models exist for many earthquakes. Although for some purposes, it may be sufficient to simply graphically compare these rupture models (e.g., Shearer and Bürgmann, 2010); in most cases, we would like to quantify and understand the differences in finite-fault rupture models (Gallovič and Ampuero, 2015; Razafindrakoto et al., 2015) and address the issue of quantifying uncertainty in source inversions (Beresnev, 2003; Page et al., 2011; Mai et al., 2016).

Therefore, it should become standard practice for authors to include files of numerical results, as well as relevant data and metadata, to allow readers to utilize the published research results for follow-up scientific work. A standardized format for disseminating models and data will also enable developing community codes that use formatted inputs for further analysis, which further facilitate transparent and transformative scientific practice, as advocated in this article. In addition, we encourage authors to facilitate access to time-series data (and, if applicable, Global Positioning System [GPS] data) such that quantitative comparisons (and related figures) can be easily generated in subsequent studies. Although this requires more initial effort by the authors, it should reduce repeated individual requests for model descriptions and documentation that often occur at present. Clearly, this practice would be encouraged if scientific publications embraced some standards in model documentation as defined below. However, at the current state of practice in source-modeling documentation, it is important to first promote a culture of making these models (and related data and documentation) easily available for further research before attempting to finalize all the details of what ultimately could be provided. Thus, we regard our proposed documentation scheme as a first step to establish an agreed upon practice and format for documenting finite-fault earthquake rupture models. The standards and formats proposed here may be revised, updated, and improved subsequently, based on the experience gathered with the proposed schemes below.

\section{DOCUMENTATION OF FINITE-FAULT INVERSIONS}

Published slip-inversion models should provide the information specified in the three sections below, related to documenting the model, the data, and the method. Although some of this information can be conveniently given in the journal publication itself, it is often advantageous to disseminate large amounts of data/tables by means of an electronic supplement or through a community database. We encourage authors to supply all of the information listed below, where items flagged with an ${ }^{*}$ are strongly recommended, but not mandatory.

\section{Model Documentation}

First, a detailed description of all relevant modeling parameters should be provided, followed by the exact space-time information of the inferred finite-fault rupture model. In particular:

1. A tabulated model of seismic $P$ and $S$ velocities, density, and attenuation parameters versus depth used to compute the Green's functions. This needs to be specified in sufficient detail such that any reader/researcher can retroactively obtain the assumed Earth structure at any given position. If applicable, provide site-specific corrections (time/amplitude), or site-specific velocity-density structures.

2. The hypocenter location (latitude, longitude, and depth) and origin time, as assumed or inferred through the source inversion process.

3. The geographic locations (latitude, longitude, and depth) of all points on the fault for which unique Green's functions are computed, and the shear modulus value used at each point for the calculations. This set of points is often denser than the set of subfaults that describe the finite-fault rupture model. If Green's functions are computed for a dislocation source acting over a small finite-size fault patch, information as specified below in item (4) should be given.

4. For each subfault: a unique index number, latitude, longitude, and depth of all corners (four for rectangular subfaults and three for triangular subfaults). This information defines the location and geometry of all subfaults in the finite-fault rupture model.

5. For each subfault: index number (defined in item 4), final slip (computed by integrating the local slip-rate function defined in item 6), rise time (slip duration), and ruptureonset time (if applicable). 
6. For each subfault: index number (defined in item 4), sliprate function (slip velocity versus time, relative to hypocentral origin time or to a specified absolute reference time), as used in generating the predicted time series. The instantaneous rake angle should also be included if it is allowed to vary in the inversion.

7. Total seismic moment. If the shear-modulus varies over the fault, potency should be reported.

8. *Any other key parameters that the author(s) consider important for understanding their model and ensuring reproducibility. These can be documented in the form of an electronic supplement to the journal publication, containing any additional material, descriptions, links to webpages, references, and related documentation that supports the published finite-fault rupture model. Detailed processing and data analysis steps taken to generate the results (see the Method Documentation section) and not specified in the journal publication can be reported here. Such an electronic supplement can be made available by various means of openly accessible electronic-data dissemination and storage (website, file transfer protocol [FTP] server, and community database).

Although the above list provides an adequate documentation for most finite-fault inversions, we make the following remarks for further explanations and regarding additional information:

1. For offshore events, the water depth should be specified, and whether depths of the hypocenter, subfaults, and so on, are given relative to the water surface or to the seafloor.

2. For teleseismic waves, attenuation parameters (e.g., $t^{*}$ values) should be provided for phases used in the inversion.

3. For 3D models or station-specific models, an average $1 \mathrm{D}$ model and a general description of the $3 \mathrm{D}$ variations should be provided (a complete description of the 3D model is not required, but reference must be made to which 3D Earth model has been used, ideally with a hyperlink to a download site for the digital version of this model).

4. Items (3) and (4) in the above list "Model Documentation" are listed separately to account for the possibility that multiple Green's functions are computed for the same subfault. The shear modulus is included in item (3) to account for the possibility of $3 \mathrm{D}$ variations not fully included in item (1).

5. Values for latitude, longitude, and depth should be provided with enough significant digits to precisely specify the fault model.

6. For flat-layered Earth models, latitude and longitude may be supplemented by spatial sampling $(d x$ and $d y)$ in kilometers relative to a provided reference location (latitude and longitude), but the author's best estimate for latitude and longitude should also be given.

7. Description of any filter applied to the Green's functions.

8. It needs to be clearly stated whether the source inversion also solves for the geometry of the rupture surface, any of its segments, the subfault size and location, or the location of individual point sources. If yes, the particular inversion approach needs to be documented. If not, it should be clearly stated which model parameters are assumed fixed, and which ones are solved for in the inversion.

9. ${ }^{*}$ If the source inversion is done using an unstructured fault model parameterization (triangular or spectral element fault meshes), a fault-mesh file could be supplied. A possible format is as follows:

- Line 1: three integers: number of subfaults (NF), number of vertices per element $(\mathrm{NV}=3$ or 4$)$, total number of vertices (NTOT).

- Next NTOT lines, one per vertex: vertex unique id, latitude, longitude, and depth (one integer and three reals).

- Next NF lines, one per subfault: subfault unique id, ids of the vertices of current subfault $(1+\mathrm{NV}$ integers). A significant part of the information listed above can be provided through existing file formats that represent finite-fault rupture models. As primary file format, we recommend the SRCMOD database format (see Data and Resources, Fig. 1). A more comprehensive format that provides the precise spacetime information of the rupture kinematics is the standard rupture format (.srf) convention proposed by Rob Graves (U.S. Geological Survey). This format specifies the exact slip-rate history on each point on the fault in three slip directions and is used, for instance, by the ground-motion simulation platform of the Southern California Earthquake Center. The detailed format description can be found in Data and Resources.

\section{Data Documentation}

In this section, a detailed description should be given of the various data (and, if applicable, data covariances) that has been used in the finite-fault inversion. In particular,

For seismic data and related processing

1. list all seismic phases modeled (e.g., $P, S, R_{1}, W$ phase, etc.);

2. list seismic stations used, with the following information for each;

- station name, channel code, and component(s);

- station location (latitude, longitude, and elevation);

- specify displacement, velocity, or acceleration;

- sample rate;

- filter band, if data are filtered, and filter parameters (causal, acausal, etc.);

- *list of seismic phases and components modeled for the station, if different from what is given in item (1) above; and

- ${ }^{*}$ relative station and/or phase weighting coefficients

3. in case an empirical Green's function approach is used, access to raw and processed data should be facilitated, along with the item (2) and its subitems above.

For geodetic data

1. list of continuous GPS stations used and related processing:

- station location (latitude, longitude, and elevation);

- source of time;

- sample rate;

- filter band, if data are filtered; and

- who processed the data and when? Which processing software used? Date of data download for the particular study?; 
(a)

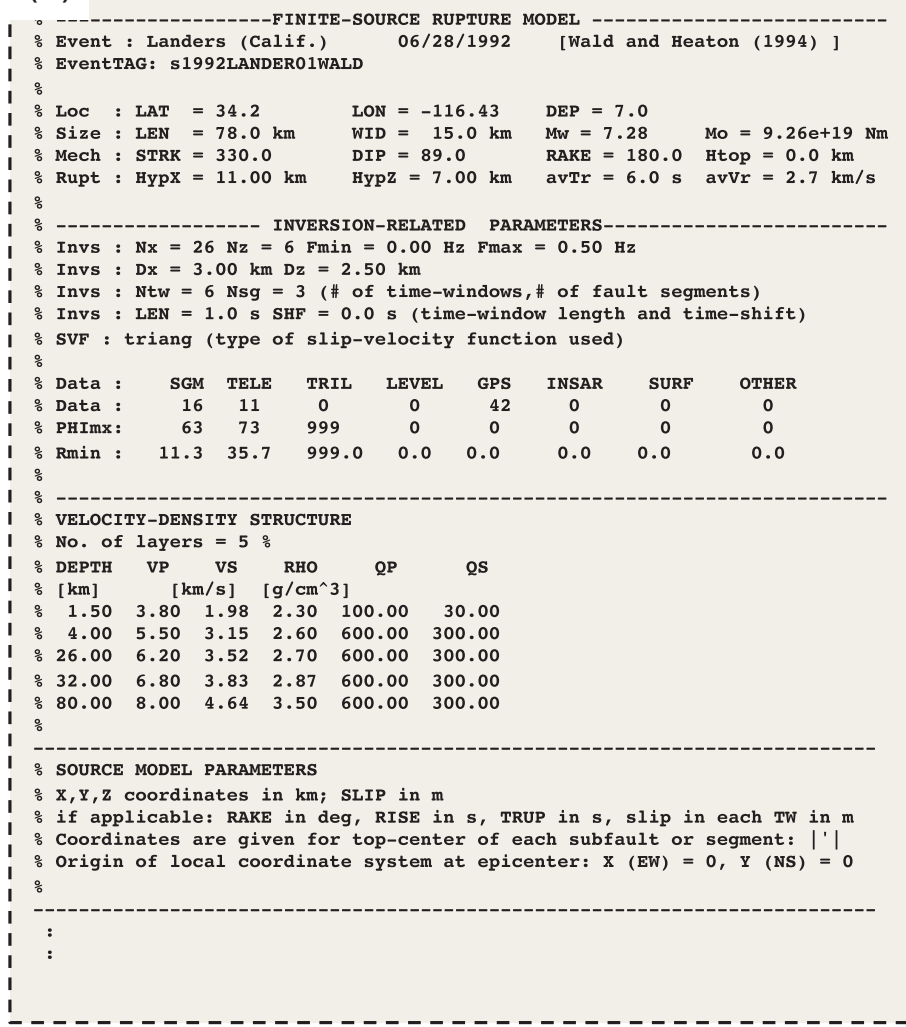

(b)

\% SEGMENT \# 2: STRIKE $=334.0 \mathrm{deg}$ DIP $=90.0 \mathrm{deg}$

I LEN $=37.00 \mathrm{~km}$ WID $=15.00 \mathrm{~km}$

I LEN $=37.00 \mathrm{~km}$ WID $=15.00 \mathrm{~km}$

I $\%$ depth to top: z2top $=0.00 \mathrm{~km} \div$ coordinates of top-center:

I LAT $=34.4150$, LON $=-116.4800$

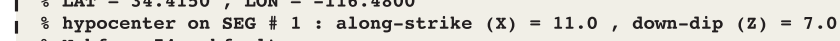

I Nsbfs $=54$ subfaults

I LAT LON $\mathrm{x}==\mathrm{EW} \quad \mathrm{y}==\mathrm{NS} \quad \mathrm{z}$ SLIP Tw1 Tw2 $\ldots$

$\begin{array}{llllllllll} & 34.3179 & -116.4228 & 0.6652 & 13.1053 & 2.5000 & 3.230 & 1.310 & 0.280 & \ldots\end{array}$

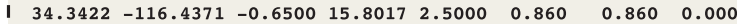

1.

$1 \%$ SEGMENT \# 1: STRIKE $=355.0 \mathrm{deg}$ DIP $=90.0 \mathrm{deg}$

I LEN $=30.00 \mathrm{~km}$ WID $=15.00 \mathrm{~km}$

I $\%$ depth to top: z2top $=0.00 \mathrm{~km} \%$ coordinates of top-center:

LAT $=34.2280$, LON $=-116.4350$

$\%$ hypocenter on SEG \# 1 : along-strike $(x)=11.0$, down-dip $(z)=7.0$ $\because \mathrm{Nsbfs}=60$ subfaults

LON $\mathrm{x}==\mathrm{EW}$ LAT $\mathrm{y}==\mathrm{NS} \quad \mathrm{z} \quad$ SLIP Tw1 Tw2 $\ldots$

:-

\begin{tabular}{l|llllllll}
34.3221 & -116.4450 & -1.3747 & 13.5714 & 2.5000 & 3.400 & 1.270 & 0.460 & $\ldots$
\end{tabular}

$\begin{array}{lllllllll}34.3490 & -116.4478 & -1.6361 & 16.5600 & 2.5000 & 5.890 & 1.300 & 1.060 & \ldots\end{array}$

:

1 (1)

$\therefore$ SEGMENT \# 3: STRIKE $=320.0 \mathrm{deg}$ DIP $=90.0 \mathrm{deg}$

1 LEN $=36.00 \mathrm{~km}$ WID $=15.00 \mathrm{~km}$

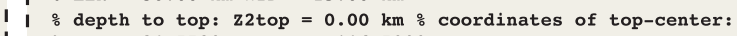

I LAT $=34.5720$, LON $=-116$.

I $\%$ hypocenter on SEG \# 1 : along-strike $(x)=11.0$, down-dip $(z)=7.0$ I $:$ Nsbfs $=72$ subfaults

$\begin{array}{llllllll}1 & : \text { LAT LON } & x==E W & y==N S & z & \text { SLIP } & \text { TW1 } & \text { TW2 }\end{array}$

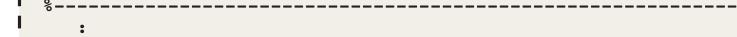

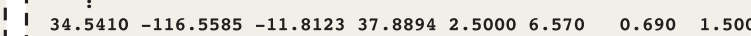

$\begin{array}{llllllll}34.5617 & -116.5795 & -13.7406 & 40.1875 & 2.5000 & 5.250 & 0.990 & 1.530\end{array}$ 1

(c)

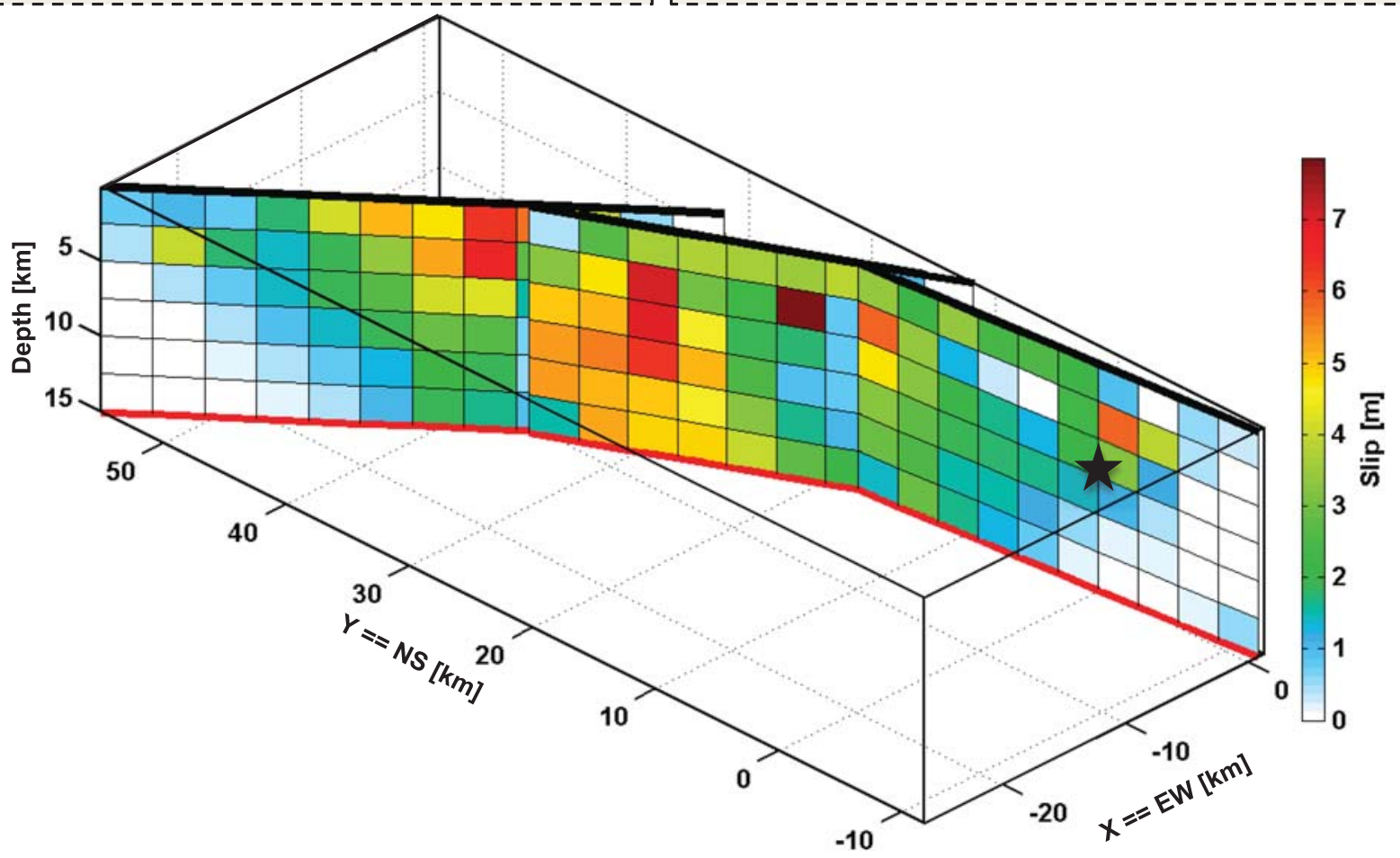

- Figure 1. The proposed finite-fault rupture model documentation. (a) List of overall earthquake source parameters, as well as metadata and information related to the inversion parameterization. (b) Documentation (abbreviated here) of the kinematic source parameters for each subfault, grouped into the three major segments of the fault model shown in (c). Subfault locations are given in latitude, longitude, and depth coordinates, as well as in a local Cartesian coordinate system with origin at the epicenter. The reference point for each subfault as well as each segment is its top center. (c) Graphical representation of the finite-fault-slip distribution described above ( $a$ and b). The star marks the hypocenter. For further details of the SRCMOD file format, please see Data and Resources. 
2. list of campaign-mode GPS stations used and related processing:

- station location (latitude, longitude, and elevation);

- source of time series; and

- who processed the data and when? Which processing software used? Date of data download for the particular study?

3. list of Interferometric Synthetic Aperture Radar (InSAR) images used and related processing:

- (latitude and longitude) of the four corner of the study region, and the respective InSAR scenes;

- satellite type/mission and recording band;

- date/time of recording and processing the InSAR scenes;

- who processed the data and when? Which processing software used? Which processing steps were applied (e.g., interferogram filtering, ramp removal, topography removal, atmospheric corrections, phase unwrapping, ambiguity estimation across ruptures, subsampling, etc.); and

- if the used InSAR data are spatially subsampled before inversion, the resulting location of subsampled pixels and corresponding surface displacements should be specified.

For tsunami data and related processing

1. list of stations used:

- station location (latitude, longitude, and elevation or depth);

- specify instrument type (tide gauge, buoy, satellite, etc.), and data type (e.g., water height, pressure, etc.);

- sample rate; and

- filter band/processing applied (e.g., tide correction method).

Parameters common to all stations can be given once, that is, they do not need to be listed separately for each station (e.g., sample rate, filter band, etc.).

In addition to the above recommendations, we urge the authors to provide:

- pointers (hyperlinks) to download sites where the raw data can be retrieved and

- access to the processed data as used in the inversion, through an electronic supplement or via a dedicated website or FTP server. If the data are not in a standard format, a description of the data format should be provided. This also applies to time-series data. Ideally, both data and model-predicted synthetics are supplied to facilitate the subsequent analysis and reproducibility.

\section{Method Documentation}

It is imperative to provide detailed descriptions of the methods for forward calculations, data processing, and data inversion that have been applied during the finite-fault inversion. Specifically, the following information should be provided:

1. Method used to compute the Green's functions:

- reference(s) to the method;

- if a publically available Green's function code was used, provide its version and a method to obtain it (e.g., a link to download it);
- if the authors developed their own code based on the given reference, describe how the method was implemented;

- describe any original modifications of the method that were introduced; and

- if the applied code is not open source, provide the Green's functions that were used in the study

2. Documentation of data-processing schemes, for example, specifying any data corrections, filtering, downsampling, and related parameters applied to the original recorded observations to generate the actual data used in the inversion.

3. General description of the inversion approach used, including definition of cost/misfit function, norms used, any applied smoothing/regularization, as well as any Bayesian priors. The particular choice of the priors should be justified, and information should be provided on how the final (optimal) smoothing/regularization parameters were selected.

4. Information on the data misfit for the preferred model. For joint inversions, ideally this would include separate misfit numbers for the different data types (e.g., seismic, geodetic, tsunami, etc.) and how the different data types were weighted in the inversion. A justification for the applied weighting scheme must be provided as well.

\section{SUMMARY}

The seismological community has been a forerunner in open global data exchange for decades, for instance through the Incorporated Research Institutions for Seismology (IRIS) Data Management System and regional and international efforts also providing freely accessible seismic and earthquake data. Open and ready access to high-level seismological data products, such as tomographic Earth models or finite-fault rupture models, still has much room for expansion. This requires access to relevant documentation of the scientific workflow, from original signals to modeling/inversion procedures to the final research results that can be used for further investigations. Here, we propose a combined documentation and formatting scheme for documenting finite-fault earthquake source inversions and the resulting kinematic rupture models. We envision that if the community embraces this approach it will facilitate reproducibility as well as interoperability of the models across the seismology and earthquake-engineering community. An agreed-upon scheme for documenting finite-fault source inversions is also a necessary step for developing new approaches for rigorous uncertainty quantification (e.g., Beresnev, 2003; Page et al., 2011; Mai et al., 2016). However, the proposed scheme is only a first step to promote common source-modeling reporting standards to foster a culture of sharing these models (and related data and information) for future research. As the seismological communities (and other scientific communities) gather experience with the proposed formats, we anticipate updates and modification to satisfy additional requirements and applications.

Finite-fault rupture models for large and great global earthquakes have a wide range of applications for subsequent research 
to understand earthquake source processes and seismotectonics, and are also of great use for seismic-hazard research applications. As inversion methods become more sophisticated, real-time data is increasingly available, and computer capabilities are enhanced, we see growing numbers of finite-fault rupture models for a given earthquake being generated by many scientific teams (often in near-real time). This motivates progressing beyond simple graphical comparisons of these rupture models, to quantitative assessments of the quality and robustness of the models. There is value in using well-defined statistical metrics to understand the similarities and discrepancies between these rupture models (Gallovič and Ampuero, 2015; Razafindrakoto et al., 2015; Zhang et al., 2015). For example, one approach is to run a posteriori forward simulations for proposed source-model solutions on a common dataset to assess the data-prediction capabilities for cross validation. Given that advanced computing will be increasingly used to deal with many, potentially very large, rupture-model datasets, it may also be necessary to move soon from ASCII-formatted earthquake-source representations to commonly used binary formats (HDF5 and NetCDF) for which standardized input/output-libraries exist. This type of forward-looking scientific cooperation and integration (potentially moving to community-consensus representations of the faulting process for critical events) can only be achieved after implementing clearly defined standards, formats, and protocols for documenting and disseminating our research. Our anticipation is that researchers quickly adopt the proposed documentation format, whereas at the same time journal editors require that authors follow these guidelines. We also encourage authors to supply their finite-fault rupture models to an online repository (e.g., the SRCMOD database, Mai and Thingbaijam, 2014) that checks the validity of the provided rupture-model data and associated information. Finally, it is important to first promote a culture of making these models (and related data and documentation) easily available for further research, before attempting to finalize all the details of what ultimately should be provided.

\section{DATA AND RESOURCES}

The finite-fault rupture model and associated data-formatting description (shown in Fig. 1) are extracted from the SRCMOD database (http://equake-rc.info/SRCMOD/fileformats/fsp and http://equake-rc.info/srcmod, last accessed December 2015). The detailed format description can be found in http://equake -rc.info/static/publish/paper/SRF-Description-Graves_2.0.pdf. Stanford Exploration Project can be found in http://sepwww. stanford.edu/doku.php. The unpublished manuscript by K. K. S. Thingbaijam and P. M. Mai (2016), "Evidence 1 for truncated exponential probability distribution of earthquake slip", submitted for publication to Bull. Seismol. Soc. Am. $\mathbf{<}$

\section{ACKNOWLEDGMENTS}

We are grateful to Rob Graves (U.S. Geological Survey [USGS]) for supporting our efforts by making his standardrupture-format (.srf) openly available. Comments by M. van
Driel, F. Pollitz, R. Okuwaki, Y. Yagi, and O. Zielke helped to refine and clarify the formatting requirements when using the srf convention for computing synthetic seismograms at teleseismic distances. S. Jónsson helped to define Interferometric Synthetic Aperture Radar (InSAR) data requirements. We thank the two reviewers, J. Murray and T. Iwata, for their valuable comments and constructive criticism. Their feedback helped to improve not only the manuscript, but also the proposed data and metadata documentation. This research was supported by the Southern California Earthquake Center (SCEC). SCEC is funded by National Science Foundation (NSF) Cooperative Agreement EAR-1033462 and USGS Cooperative Agreement G12AC20038. The SCEC Contribution Number for this article is 2107 . The research presented in this article is supported by the Division of Physical Sciences and Engineering, King Abdullah University of Science and Technology (KAUST).

\section{REFERENCES}

Agnew, D. C. (2012). AGU journals should ask authors to publish results, Eos Trans. $A G U$ 93, no. 29, 266.

Agnew, D. C. (2013). Realistic simulations of geodetic network data; the Fakenet package, Seismol. Res. Lett. 84, 426-432.

Beresnev, I. A. (2003). Uncertainties in finite-fault slip inversions: To what extent to believe? (A critical review), Bull. Seismol. Soc. Am. 93, 2445-2458.

Causse, M., F. Cotton, and P. M. Mai (2010). Constraining the roughness degree of slip heterogeneity, J. Geophys. Res. 115, no. B05304, doi: 10.1029/2009JB006747.

Causse, M., L. A. Dalguer, and P. M. Mai (2013). Variability of dynamic source parameters inferred from kinematic models of past earthquakes, Geophys. J. Int. 196, 1754-1769, doi: 10.1093/gji/ggt478.

Eddy, D. M., W. Hollingworth, J. J. Caro, J. Tsevat, K. M. McDonald, and J. B. Wong (2012). Model transparency and validation: A report of the ISPOR-SMDM modeling good research practices task force-7, Med. Decis. Making 32, 733-743.

Ergintav, S., S. McClusky, E. Hearn, R. Reilinger, R. Cakmak, T. Herring, H. Ozener, O. Lenk, and E. Tari (2009). Seven years of postseismic deformation following the $1999, \mathrm{M}=7.4$ and $\mathrm{M}=7.2$, IzmitDüzce, Turkey earthquake sequence, J. Geophys. Res. 114, no. B07403, doi: 10.1029/2008JB006021.

Gabriel, A.-A., J.-P. Ampuero, L. A. Dalguer, and P. M. Mai (2012). The transition of dynamic rupture styles in elastic media under velocityweakening friction, J. Geophys. Res. 117, no. B09311, doi: 10.1029/ 2012JB009468.

Gallovič, F., and J.-P. Ampuero (2015). A new strategy to compare inverted rupture models exploiting the Eigen-structure of the inverse problem, Seismol. Res. Lett. 86, 1679-1689.

Haak, L. L., D. Baker, D. K. Ginther, G. J. Gordon, M. A. Probus, N. Kannankutty, and B. A. Weinberg (2012). Standards and infrastructure for innovation data exchange, Science 338, no. 6104, 196-197, doi: 10.1126/science.1221840.

Hanson, B., A. Sugden, and B. Alberts (2011). Making data maximally available, Science 331, no. 6018, 649, doi: 10.1126/science.1203354.

Heaton, T. H. (1990). Evidence for and implications of self-healing pulses of slip in earthquake rupture, Phys. Earth Planet. In. 64, 1-20.

Ide, S., and M. Takeo (1997). Determination of constitutive relations of fault slip based on seismic waves analysis, J. Geophys. Res. 102, 27,379-27,391.

Lavallée, D., P. Liu, and R. J. Archuleta (2006). Stochastic model of heterogeneity in earthquake slip spatial distributions, Geophys. J. Int. 165, 622-640. 
Lees, J. M. (2012). Open and free: Software and scientific reproducibility, Seismol. Res. Lett. 83, no. 5, 751-752, doi: 10.1785/0220120091.

Mai, P. M., and G. C. Beroza (2000). Source-scaling properties from finite-fault rupture models, Bull. Seismol. Soc. Am. 90, 604-615.

Mai, P. M., and G. C. Beroza (2002). A spatial random-field model to characterize complexity in earthquake slip, J. Geophys. Res. 107, no. B11, 2308, doi: 10.1029/2001JB000588.

Mai, P. M., and K. K. S. Thingbaijam (2014). SRCMOD: An online database of finite source rupture models, Seismol. Res. Lett. 85, no. 6, 1348-1357, doi: 10.1785/0220140077.

Mai, P. M., D. Schorlemmer, M. Page, J.-P. Ampuero, K. Asano, M. Causse, S. Custodio, W. Fan, G. Festa, M. Galis, et al. (2016). The earthquake-source inversion validation (SIV) project, Seismol. Res. Lett. 87, no. 3, doi: 10.1785/0220150231.

Mai, P. M., P. Somerville, A. Pitarka, L. Dalguer, H. Miyake, G. Beroza, S.-G. Song, and K. Irikura (2006). On scaling of fracture energy and stress drop in dynamic rupture models: Consequences for nearsource ground-motions, in Earthquakes: Radiated Energy and the Physics of Faulting, R. Abercrombie, A. McGarr, G. Di Toro, and H. Kanamori (Editors), Vol. 170, American Geophysical Union, Washington, D. C., 283-294.

Mai, P. M, P. Spudich, and J. Boatwright (2005). Hypocenter locations in finite-source rupture models, Bull. Seismol. Soc. Am. 95, 965-980.

Manighetti, I., M. Campillo, C. Sammis, P. M. Mai, and G. King (2005). Evidence for self-similar, triangular slip distributions on earthquakes: Implications for earthquake and fault mechanics, J. Geophys. Res. 110, no. B05302, doi: 10.1029/2004JB003174.

Mesirov, J. P. (2010). Accessible reproducible research, Science 327, no. 5964, 415-416, doi: 10.1126/science.1179653.

Page, M., P. M. Mai, and D. Schorlemmer (2011). Testing earthquake source inversion methodologies, Eos Trans. $A G U$ 92, no. 9, 75.

Peng, R. D. (2011). Reproducible research in computational science, Science 334, no. 6060, 1226-1227, doi: 10.1126/science.1213847.

Razafindrakoto, H. N. T, P. M. Mai, M. G. Genton, L. Zhang, and K. K. S. Thingbaijam (2015). Quantifying variability in earthquake rupture models using multidimensional scaling: Application to the 2011 Tohoku earthquake, Geophys. J. Int. 202, 17-40, doi: 10.1093/ gji/ggv088.

Schwab, M., M. Karrenbach, and J. Claerbout (2000). Making scientific computations reproducible, Comput. Sci. Eng. 2, no. 6, 61-67.

Shearer, P. M., and R. Bürgmann (2010). Lessons learned from the 2004 Sumatra-Andaman megathrust rupture, Annu. Rev. Earth Planet. Sci. 38, 103-131, doi: 10.1146/annurev-earth-040809-152537.

Somerville, P., K. Irikura, R. Graves, S. Sawada, D. Wald, N. Abrahamson, Y. Iwasaki, T. Kagawa, N. Smith, and A. Kowada (1999). Character- izing crustal earthquake slip models for the prediction of strong ground motion, Seismol. Res. Lett. 70, 59-80.

Stein, R. S. (2003). Earthquake conversations, Sci. Am. 288, 72-79.

Strasser, F. O., M. C. Arango, and J. J. Bommer (2010). Scaling of the source dimensions of interface and intraslab subduction-zone earthquakes with moment magnitude. Seismol. Res. Lett. 81, 941-950.

Tinti, E., P. Spudich, and M. Cocco (2005). Earthquake fracture energy inferred from kinematic rupture models on extended faults, J. Geophys. Res. 110, no. B12303, doi: 10.1029/2005JB003644.

Zhang, L., P. M. Mai, K. K. S. Thingbaijam, H. N. Razafindrakoto, and M. Genton (2015). Comparing earthquake slip models with the Spatial Prediction Comparison Test, Geophys. J. Int. 200, no. 1, 185-198, doi: 10.1093/gji/ggu383.

\section{P. Martin Mai}

King Abdullab University of Science and Technology Division of Physical Sciences and Engineering Thuwal 23955-6900, Kingdom of Saudi Arabia martin.mai@kaust.edu.sa

Peter Shearer
University of California, San Diego
IGPP 0225

La Jolla, California 92093 U.S.A.

Jean-Paul Ampuero

California Institute of Technology Seismological Laboratory

1200 East California Boulevard, MC 252-21 Pasadena, California 91125 U.S.A.

Thorne Lay

University of California Santa Cruz

Department of Earth and Planetary Sciences 1156 High Street Santa Cruz, California 95064 U.S.A.

Published Online 6 April 2016 